\title{
A Food, a Bite, a Sip: How Much Allergen Is in That?
}

\author{
Melanie Kok ${ }^{1}$, Astrid Compagner ${ }^{1}$, Ina Panneman ${ }^{2}$, Aline Sprikkelman ${ }^{3}$ and Berber Vlieg-Boerstra ${ }^{1,4, *(\mathbb{D})}$ \\ 1 Department of Nutrition and Dietetics, Hanze University of Applied Sciences, \\ 9713 GZ Groningen, The Netherlands; melaniekok02@hotmail.com (M.K.); \\ astrid_compagner@hotmail.com (A.C.) \\ 2 The Food College, Practice for Diet, Nutrition, Sports and Food Technology, 8651 AE IJlst, The Netherlands; \\ hetvoedingscollege@online.nl \\ 3 Department of Pediatric Pulmonology and Allergy, University Medical Center Groningen, University of \\ Groningen, 9700 RB Groningen, The Netherlands; a.b.sprikkelman@umcg.nl \\ 4 Department of Paediatrics, OLVG Hospital, 1090 HM Amsterdam, The Netherlands \\ * Correspondence: b.vlieg-boerstra@olvg.nl; Tel.: +31-62-096-5612
}

\section{check for}

updates

Citation: Kok, M.; Compagner, A.; Panneman, I.; Sprikkelman, A.; Vlieg-Boerstra, B. A Food, a Bite, a Sip: How Much Allergen Is in That? Nutrients 2021, 13, 587. https://doi.org/10.3390/nu13020587

Academic Editor: Linda Monaci

Received: 13 January 2021

Accepted: 2 February 2021

Published: 10 February 2021

Publisher's Note: MDPI stays neutral with regard to jurisdictional claims in published maps and institutional affiliations.

Copyright: (c) 2021 by the authors. Licensee MDPI, Basel, Switzerland. This article is an open access article distributed under the terms and conditions of the Creative Commons Attribution (CC BY) license (https:/ / creativecommons.org/licenses/by/ $4.0 /)$.

\begin{abstract}
Detailed information about the amount of allergenic protein ingested by the patient prior to an allergic reaction yields valuable information for the diagnosis, guidance and management of food allergy. However, the exact amount of ingredients is often not declared on the label. In this study the feasibility was studied for estimating the amount of allergenic protein from milk, eggs, peanuts and hazelnuts in frequently consumed composite and non-composite foods and per bite or sip size in different age groups in the Netherlands. Foods containing milk, egg, peanut or hazelnut most frequently consumed were selected for the age groups 2-3, 4-6 and 19-30 years. If the label did not yield clear information, the amount of allergenic protein was estimated based on food labels. Bite or sip sizes were determined in these age groups in 30 different foods. The amount of allergenic protein could be estimated in 47/70 (67\%) of composite foods, which was complex. Estimated protein content of milk, egg, peanut and hazelnut was 2-3 g for most foods but varied greatly from 3 to $8610 \mathrm{mg}$ and may be below threshold levels of the patient. In contrast, a single bite or sip can contain a sufficient amount of allergenic protein to elicit an allergic reaction. Bite and sip sizes increased with age. In every day practice it is hard to obtain detailed and reliable information about the amount of allergenic protein incorporated in composite foods. We encourage companies to disclose the amount of common allergenic foods on their labels.
\end{abstract}

Keywords: diet history; food allergy; allergenic protein; thresholds; eliciting dose; bite size; cow's milk; hen's egg; peanut; hazelnut

\section{Introduction}

For health care professionals who are involved in food allergies, detailed information about the amount of allergenic protein ingested by the patient prior to an allergic reaction yields valuable information for the diagnosis, guidance and management of the food allergy. This information can be obtained by a detailed allergy-focused diet history. One of the aims of the diet history in allergies is to identify suspected foods by linking symptoms to foods [1-4]. Information about the type and amount ingested which elicited allergic reactions, as well as the severity of the reaction, helps to estimate the clinical sensitivity of the patient and the risk for severe reactions. It is generally accepted that the higher the amount ingested, the more severe the expected allergenic reaction [1,5]. A low eliciting dose is assumed to reflect a higher clinical sensitivity [6] and may therefore be an indication for prescription of an epinephrine auto-injector [7]. This is important information for the design of the oral food challenge test for diagnosis and may lead to more stringent dietary advice. In contrast, in certain patients a high eliciting dose may lead to less stringent dietary advice [8]. 
The amount of allergenic protein ingested should be estimated by the health care professional from both the portion size ingested and from the amount of allergenic protein present in the food [2,3].

Often the patient has not consumed a full portion of a food but may only have taken one or a few bites or sips from the food until the reaction developed. Thus, in that case the health care professional should estimate the amount of allergenic protein ingested from the size of the bite or sip taken from the food. To our knowledge, no studies have been performed on the bite or sip sizes of foods containing allergenic ingredients.

Secondly, the health care professional should estimate the amount of allergenic protein in the food ingested [2,3]. However, in the majority of composite foods in which the protein content is delivered by several allergenic and non-allergenic ingredients, the exact amount of ingredients is not declared on the label.

The presence of fourteen major food allergens should be fully disclosed on the label in clear wordings according to European regulations. These are milk (including lactose), egg, soy, peanut, tree nuts, gluten, fish, shellfish, mollusks, celery, mustard, lupin, sesame and sulphite [9]. Risk-based approaches to managing allergens in foods are currently being developed by the food industry and regulatory authorities to support food-allergic consumers to avoid ingesting their problem food [10,11]. In non-composite foods or foods having only one protein source, the amount of allergenic ingredients can be derived from the label, e.g., milk contains 3.5\% protein from cow's milk. However, in composite foods most labels do not yield information on the amount of allergenic ingredients unless explicitly stated (e.g., Nutella contains 13\% hazelnuts). Thus, most foods lack these data which does not allow the physician or dietitian to accurately estimate the amount of allergenic protein ingested prior to an allergic reaction.

Oral food challenges are the preferred test to establish the diagnosis of food allergy $[1,2,4,12]$. During oral food challenges, the suspected food is administered to the patient in incrementing amounts with 15-20 minutes time intervals in an open, single-blind or double-blind fashion. Inter-individual thresholds to food allergens widely differ between patients, for reasons not yet fully understood. Patients may react to tiny amounts, such as crumbs of peanut or egg, or to higher doses up to full portions of the allergenic food. Therefore, 6 to 8 dose incremental scales in oral food challenges range from $1 \mathrm{mg}$ protein to more than $4 \mathrm{~g}$ protein of the allergenic food, reflecting a full portion size [1,2,12]. Information about the clinical sensitivity of the patient is important for the design of the oral food challenge. Reactions to small amounts in history require increased safety measures during oral food challenges, such as selection of the challenge setting and a lower starting dose [12].

The oral food challenge yields information about the threshold, i.e., the amount of allergenic food eliciting symptoms, as well as the severity of symptoms, although it is recognized that threshold levels in oral food challenges in a clinical setting may be different from threshold levels in everyday life and may not be reproducible [13]. It was recently shown that co-factors such as lack of sleep and physical exercise significantly decrease threshold levels [14].

Once the threshold dose in an oral food challenge is established, insight in the amount of allergenic protein in foods could allow patients with mild symptoms and a high threshold level to expand their diets with foods containing small amounts well below their threshold levels in the absence of known co-factors. However, lack of this information does not allow the dietitian or patient to select foods with allergenic protein below their thresholds to expand the diet of the patient. So far, a more practical approach has been chosen, for example in patients who have passed a baked milk or baked egg challenge. These patients are advised to introduce foods with milk or egg listed as the third ingredient on the label or further down the list [15]. Alternatively, recipes are provided by dietitians to cook or bake their own products with the tolerated amounts of protein incorporated in the recipe [15].

The aims of this study were (1) to study if it is feasible to estimate the amount of allergenic protein from milk, egg, peanut and hazelnut in frequently consumed composite 
and non-composite foods per portion, per100 $\mathrm{g}$ food, and per bite or sip size in different age groups in the Netherlands, and (2) to discuss why it is important to have detailed information of the amount of allergenic protein in foods in the diagnosis and management of food allergies.

\section{Materials and Methods}

\subsection{The Amount of Allergenic Protein in Foods}

Based on the Dutch National Food Consumption Survey 2011, the most frequently consumed foods were selected for the assessment of the amount of allergenic protein [16,17]. Foods containing milk, egg, peanut or hazelnut consumed by $1 \%$ or more of consumers in the age groups 2-3 years, 4-6 years and 19-30 years were selected. Subsequently, the amount of allergenic protein in the selected foods was estimated according to an algorithm (Figure 1), including different methods.

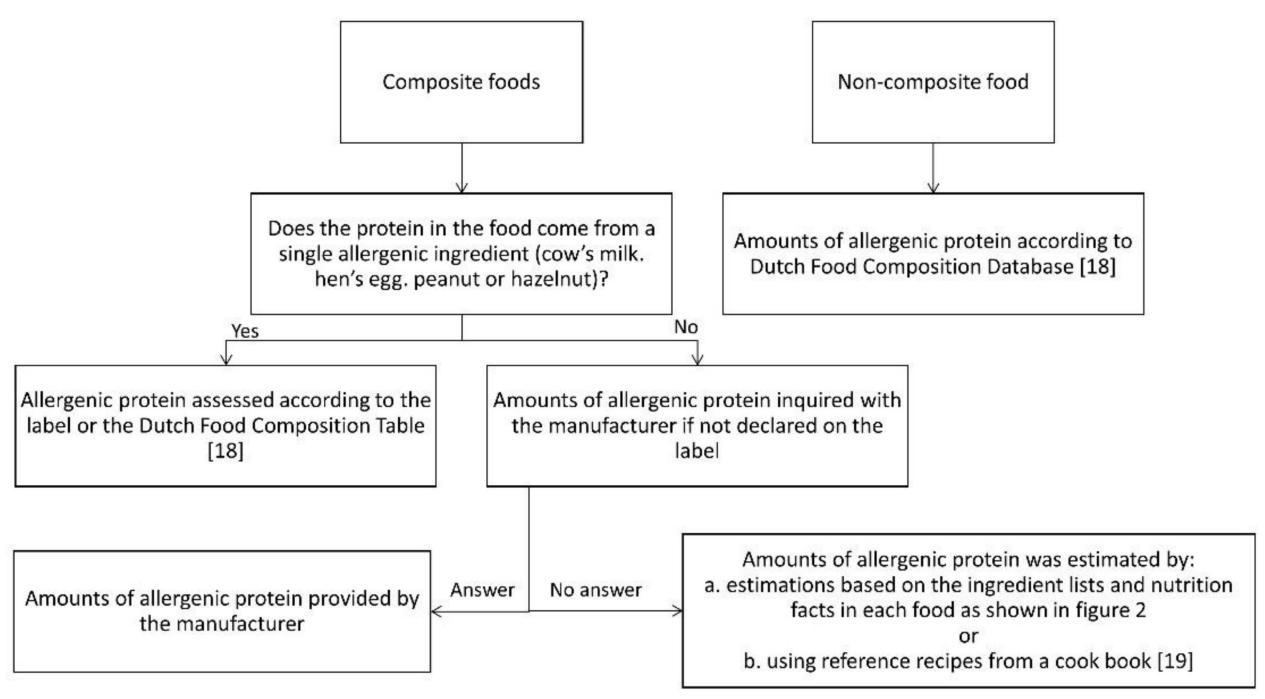

Figure 1. Assessment of the amount of allergenic protein [18,19].

In this study we defined non-composite foods as foods with only one ingredient or having only one ingredient yielding protein. We defined composite foods as foods with multiple ingredients yielding protein.

For non-composite foods, data were derived from the Dutch Food Composition Database 2011 (NEVO) [18] or food labels. For composite foods, the labels were checked for declaration of the amount of allergenic ingredients. If this was not declared, the manufacturer was contacted. When the required information was not provided by the manufacturer, the amount of allergenic protein was, as a non-validated method, estimated by calculations based on the ingredients lists and nutrition facts as follows (Figure 2):

First, it is a given fact that the ingredients on the label are listed in descending order according to their predominance by weight. Second, the nutrition facts (protein, fat, carbohydrates, energy) for each ingredient were relisted per $100 \mathrm{~g}$. Third, the nutrition facts per $100 \mathrm{~g}$ were complete when the amount of ingredients was specified on the label (e.g., $13 \mathrm{~g}$ of hazelnut, indicated in yellow in Figure 2). Fourth, the amount of the other ingredients was estimated by trial and error until, fifth, the sum of the macronutrients of the ingredients approximated the nutrition facts on the label as closely as possible. Finally, if this method was not feasible, the amount of estimated allergenic protein was based on reference recipes from a Dutch cookbook [19]. 


\begin{tabular}{|c|c|c|c|c|c|c|c|}
\hline \multicolumn{8}{|c|}{ Nutella } \\
\hline \multirow{3}{*}{ 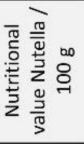 } & & \multicolumn{4}{|c|}{ Nutritional value according to label } & \multicolumn{2}{|c|}{ Result calculation } \\
\hline & & Protein $(\mathrm{g}) / 100 \mathrm{~g}$ & Carbohydrates (g) / $100 \mathrm{~g}$ & Fat $(\mathrm{g}) / 100 \mathrm{~g}$ & Kcal / $100 \mathrm{~g}$ & $\begin{array}{l}\text { Cow's milk (g) / } \\
100 \text { gram }\end{array}$ & $\begin{array}{l}\text { Hazelnut (g)/ } \\
100 \text { gram }\end{array}$ \\
\hline & & 6.0 & 57.6 & 31.6 & 546 & 1.8 & $3.2(2.3+0.9)$ \\
\hline \multirow{9}{*}{ 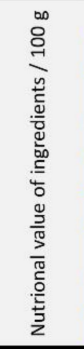 } & Ingredients & Protein $(\mathrm{g}) / 100 \mathrm{~g}$ & Carbohydrates (g) / $100 \mathrm{~g}$ & Fat $(\mathrm{g}) / 100 \mathrm{~g}$ & Kcal / $100 \mathrm{~g}$ & \multicolumn{2}{|l|}{ Reference } \\
\hline & Sugar & 0.0 & 100.0 & 0.0 & 400 & \multicolumn{2}{|l|}{ [18] } \\
\hline & Vegetable oil & 0.0 & 0.0 & 100.0 & 900 & & \\
\hline & Hazelnuts & 14.0 & 6.0 & 69.0 & 717 & \multicolumn{2}{|l|}{$[18]$} \\
\hline & Low fat cacao & 19.8 & 10.8 & 24.5 & 236 & \\
\hline & Skimmed milk powder & 35.0 & 50.0 & 1.0 & 349 & \\
\hline & Whey powder & 85.0 & 10.0 & 5.0 & 425 & \\
\hline & Lecithins & 10.0 & 35.0 & 50.0 & 620 & \\
\hline & Vanilla & & & & & & \\
\hline \multirow{10}{*}{ 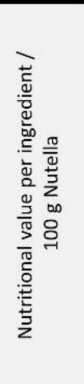 } & Ingredients & Protein $(\mathrm{g}) / 100 \mathrm{~g}$ & Carbohydrates (g) / $100 \mathrm{~g}$ & Fat $(\mathrm{g}) / 100 \mathrm{~g}$ & Kcal / $100 \mathrm{~g}$ & \multicolumn{2}{|l|}{ Ingredients (g) } \\
\hline & Sugar & 0.0 & 46.0 & 0.0 & 184 & \multicolumn{2}{|l|}{46.0} \\
\hline & Vegetable oil & 0.0 & 0.0 & 20.0 & 180 & \multicolumn{2}{|l|}{20.0} \\
\hline & Hazelnuts & 1.8 & 0.8 & 9.0 & 93 & \multicolumn{2}{|l|}{13.0} \\
\hline & Low fat cacao & 1.5 & 7.4 & 1.8 & 18 & \multicolumn{2}{|l|}{7.4} \\
\hline & Skimmed milk powder & 2.3 & 3.3 & 0.1 & 23 & \multicolumn{2}{|l|}{6.6} \\
\hline & Whey powder & 0.9 & 0.1 & 0.1 & 4 & \multicolumn{2}{|l|}{1.0} \\
\hline & Lecithins & 0.1 & 0.2 & 0.3 & 3 & \multicolumn{2}{|l|}{0.5} \\
\hline & Vanilla & & & & & \multicolumn{2}{|l|}{0.5} \\
\hline & Total & 6.6 & 57.8 & 31.3 & 505 & \multicolumn{2}{|l|}{95.0} \\
\hline
\end{tabular}

\begin{tabular}{|c|}
\hline Procedure \\
\hline 1. Listed in descending order \\
\hline $\begin{array}{l}\text { 2. Nutritional value of } \\
\text { ingredients / } 100 \text { gram }\end{array}$ \\
\hline 3. Amount specified on label \\
\hline $\begin{array}{l}\text { 4. Estimation of remaining } \\
\text { ingredients by trial and error } \\
\text { until best of totals }\end{array}$ \\
\hline $\begin{array}{l}\text { 5. Total nutritional value } \\
\text { according to label }\end{array}$ \\
\hline $\begin{array}{l}\text { 6. Result: Estimated amount of } \\
\text { allergenic protein }\end{array}$ \\
\hline
\end{tabular}

Figure 2. Estimation of the amount of allergen from the label [18].

Following these assessments, manufacturers were contacted to verify the results of the assessment of the amount of allergenic protein and were requested to comment on our findings.

Results were compared with ED10 and ED50 values for milk, egg, peanut and hazelnut as established in Dutch children and adults by Blom et al. and Klemans et al. [20,21]. ED10 and ED50 is the amount of allergenic protein to which, respectively, $10 \%$ and $50 \%$ of the allergic subjects react with objective symptoms.

\subsection{Assessment of Bite and Sip Sizes in Different Age Groups}

\subsubsection{Selection of Foods}

For the three age groups, the top $1 \%$ of the most frequently consumed foods containing milk, egg, peanut or hazelnut, as established by the National Food Consumption Survey [16,17], were selected and were allocated into food groups. The four foods most frequently used from each of the food groups were selected for the assessment of bite and sip sizes.

\subsubsection{Study Population and Measurements of Bites and Sips}

Healthy 2 to 3-year-old children from a preschool, 4 to 6-year-old children from two primary schools, and 19 to 30-year-old students in a nutrition and dietetics faculty were included. Study participants with a food allergy or other conditions that could affect the food intake were excluded.

In the 2-3-year-old children, foods were administered to the children for a bite or sip one by one while playing games. The 4-6-year-old children were asked to take a bite or sip without any instruction and without emphasis on this task to mimic regular bite and sip sizes as closely as possible. Each food was tested in 2-19 individuals in each age group. Each child received a maximum of eight foods. The adults were informed about the purpose of the study and were asked to take a single bite or sip of the food. The adults were asked to test all foods. The food was weighed before and after every bite or sip. 


\subsubsection{Pilot Study}

A pilot study was performed prior to the study at the preschool and in one of the primary schools to test the feasibility of children taking bites or sips. The following essential findings were included in our methods: (1) to keep the attention of the children, all the foods were displayed on site to speed up the process; (2) to ensure a good appetite, the study was performed just before lunch or dinner time; and (3) to imitate the natural meal setting. The study was performed in subgroups of 4-6-year-old children.

\subsubsection{Statistics}

The results of the study were processed in SPSS. For each food the median intake was calculated in the different age groups and compared using the Mann-Whitney test, as well as the differences in intake between men and women in each age category.

\section{Results}

\subsection{The Amount of Allergenic Protein in Foods}

Ninety-seven foods were selected: 27 non-composite foods for which the amount of allergenic protein was determined using the Dutch NEVO Database [18] or the label (Table 1), and 70 composite foods (Table 2).

Table 1. Amount of estimated allergenic protein in most frequently consumed non-composite foods in $\mathrm{mg}$ or $\mathrm{ml}$ per portion, $\mathrm{mg}$ or $\mathrm{ml}$ per $100 \mathrm{~g}$ and $\mathrm{mg}$ or $\mathrm{ml}$ per median bite or sip size in different age groups.

\begin{tabular}{|c|c|c|c|c|c|c|}
\hline Food & $\begin{array}{c}\text { Composite or } \\
\text { Non-Composite Food }\end{array}$ & $\begin{array}{l}\text { Amount of } \\
\text { Protein (mg or } \\
\text { ml/Portion) }\end{array}$ & $\begin{array}{l}\text { Amount of } \\
\text { Protein (mg or } \\
\text { ml)/100g) }\end{array}$ & $\begin{array}{c}\text { Amount of Protein } \\
\text { (mg or ml)/Median } \\
\text { Bite or Sip Size } \\
\text { 2-3 Years }\end{array}$ & $\begin{array}{c}\text { Amount of Protein } \\
\text { (mg or ml)/Median } \\
\text { Bite or Sip Size } \\
\text { 4-6 years }\end{array}$ & $\begin{array}{c}\text { Amount of Protein } \\
\text { (mg or ml)/Median } \\
\text { Bite or Sip Size } \\
19-30 \text { years) }\end{array}$ \\
\hline \multicolumn{7}{|c|}{ COW'S MILK } \\
\hline \multicolumn{7}{|c|}{ Cheese } \\
\hline Cottage cheese [18] & Non-composite food & 1680 & 11,200 & 100 & 100 & 290 \\
\hline $\begin{array}{l}\text { Goat cheese } \\
\text { fresh [18] }\end{array}$ & Non-composite food & 2010 & 13,400 & 120 & 120 & 350 \\
\hline $\begin{array}{l}\text { Cheese spread } \\
20+[18]\end{array}$ & Non-composite food & 2550 & 17,000 & 150 & 150 & 440 \\
\hline Brie $60+[18]$ & Non-composite food & 3400 & 17,000 & $\mathrm{Nd}$ & $\mathrm{Nd}$ & $\mathrm{Nd}$ \\
\hline $\begin{array}{l}\text { Goat cheese } \\
\text { hard [18] }\end{array}$ & Non-composite food & 4480 & 22,400 & 250 & 250 & 760 \\
\hline $\begin{array}{c}\text { Gouda cheese } \\
48+[18]\end{array}$ & Non-composite food & 4560 & 22,800 & 250 & 250 & 780 \\
\hline $\begin{array}{l}\text { Gouda cheese 20+ } \\
\text { (low-fat) [18] }\end{array}$ & Non-composite food & 6840 & 34,200 & 380 & 380 & 1160 \\
\hline \multicolumn{7}{|c|}{ Milk, Milk Products, Milk Replacers and Ice Cream } \\
\hline $\begin{array}{c}\text { Coffee creamer, } \\
\text { powder, low-fat [18] }\end{array}$ & Non-composite food & 50 & 2000 & $\mathrm{Nd}$ & $\mathrm{Nd}$ & $\mathrm{Nd}$ \\
\hline $\begin{array}{l}\text { Coffee creamer full } \\
\text { fat [18] }\end{array}$ & Non-composite food & 50 & 8100 & $\mathrm{Nd}$ & $\mathrm{Nd}$ & $\mathrm{Nd}$ \\
\hline $\begin{array}{l}\text { Whipping } \\
\text { cream [18] }\end{array}$ & Non-composite food & 230 & 2300 & $\mathrm{Nd}$ & $\mathrm{Nd}$ & $\mathrm{Nd}$ \\
\hline Crème fraiche [18] & Non-composite food & 330 & 2200 & $\mathrm{Nd}$ & $\mathrm{Nd}$ & $\mathrm{Nd}$ \\
\hline $\begin{array}{l}\text { Crème fraiche } \\
\text { demi [18] }\end{array}$ & Non-composite food & 450 & 3000 & $\mathrm{Nd}$ & $\mathrm{Nd}$ & $\mathrm{Nd}$ \\
\hline Sour cream [18] & Non-composite food & 470 & 3100 & $\mathrm{Nd}$ & $\mathrm{Nd}$ & $\mathrm{Nd}$ \\
\hline $\begin{array}{l}\text { Fromage frais full } \\
\text { fat } 8.2 \%[18]\end{array}$ & Non-composite food & 1420 & 7100 & $\mathrm{Nd}$ & $\mathrm{Nd}$ & $\mathrm{Nd}$ \\
\hline $\begin{array}{c}\text { Fromage frais } \\
\text { low-fat } 0.5 \% \text { [18] }\end{array}$ & Non-composite food & 2020 & 10,100 & 610 & 610 & 1370 \\
\hline $\begin{array}{l}\text { Fromage frais half } \\
\text { fat } 4.6 \% \text { [18] }\end{array}$ & Non-composite food & 2300 & 11,500 & 690 & 690 & 1560 \\
\hline $\begin{array}{l}\text { Nutrilon } 2 \text { Infant } \\
\text { Formula (Nutricia) }\end{array}$ & Non-composite food & 2800 & 1400 & $\mathrm{Nd}$ & $\mathrm{Nd}$ & $\mathrm{Nd}$ \\
\hline
\end{tabular}


Table 1. Cont.

\begin{tabular}{|c|c|c|c|c|c|c|}
\hline Food & $\begin{array}{c}\text { Composite or } \\
\text { Non-Composite Food }\end{array}$ & $\begin{array}{l}\text { Amount of } \\
\text { Protein (mg or } \\
\text { ml/Portion) }\end{array}$ & $\begin{array}{l}\text { Amount of } \\
\text { Protein (mg or } \\
\text { ml)/100g) }\end{array}$ & $\begin{array}{c}\text { Amount of Protein } \\
\text { (mg or ml)/Median } \\
\text { Bite or Sip Size } \\
\text { 2-3 Years }\end{array}$ & $\begin{array}{c}\text { Amount of Protein } \\
\text { (mg or ml)/Median } \\
\text { Bite or Sip Size } \\
\text { 4-6 years }\end{array}$ & $\begin{array}{c}\text { Amount of Protein } \\
\text { (mg or ml)/Median } \\
\text { Bite or Sip Size } \\
\text { 19-30 years) }\end{array}$ \\
\hline $\begin{array}{l}3.5 \% \text { Full fat } \\
\text { yoghurt [18] }\end{array}$ & Non-composite food & 5550 & 3700 & 220 & 220 & 500 \\
\hline $\begin{array}{c}\text { Yoghurt, low-fat } \\
0.3 \%[18]\end{array}$ & Non-composite food & 6150 & 4100 & 250 & 250 & 560 \\
\hline $\begin{array}{c}\text { Yoghurt, low-fat } \\
1.5 \%[18]\end{array}$ & Non-composite food & 6750 & 4500 & 270 & 270 & 610 \\
\hline Buttermilk [18] & Non-composite food & 7500 & 3000 & $\mathrm{Nd}$ & $\mathrm{Nd}$ & $\mathrm{Nd}$ \\
\hline $\begin{array}{c}\text { Whole Milk } \\
3.5 \% \text { [18] }\end{array}$ & Non-composite food & 8250 & 3300 & 83 & 264 & 1056 \\
\hline $\begin{array}{l}\text { Semi skimmed milk } \\
1.5 \%[18]\end{array}$ & Non-composite food & 8500 & 3400 & 85 & 274 & 1088 \\
\hline $\begin{array}{c}\text { Skimmed milk } \\
0.1 \%[18]\end{array}$ & Non-composite food & 9250 & 3700 & 93 & 296 & 1184 \\
\hline \multicolumn{7}{|c|}{ Fat, Oil and Sauce } \\
\hline Butter, salted [18] & Non-composite food & 40 & 700 & $<10$ & $<10$ & $<10$ \\
\hline \multicolumn{7}{|c|}{ HEN'S EGG } \\
\hline \multicolumn{7}{|c|}{ Egg } \\
\hline Boiled egg [18] & Non-composite food & 6200 & 12,300 & 308 & 615 & 1476 \\
\hline \multicolumn{7}{|c|}{ PEANUT } \\
\hline \multicolumn{7}{|c|}{ Spread } \\
\hline $\begin{array}{l}\text { Peanut butter } \\
\text { (Calvé) }\end{array}$ & Non-composite food & 3200 & 21,420 & 210 & 190 & 560 \\
\hline
\end{tabular}

mg, milligram; ml, milliliter; g, gram; Nd, no data.

For these 70 composite foods, 37 different food manufacturers and two supermarket chains were contacted by telephone and email. Only four different manufacturers provided the required data for four foods.

The amount of allergenic protein of the remaining 66 foods was estimated by the method depicted in Figure 2. The amount of allergenic protein could be estimated in 47/70 $(67 \%)$ of the composite foods and are listed in Table 2. In 15/47 (32\%) of the included composite foods, at least one allergenic ingredient was quantified on the label (e.g., Nutella, $13 \%$ hazelnut). For $19 / 70$ (27\%) of the composite foods, it was unfeasible to assess the amount of allergenic protein because the nutritional value of the main ingredients could not be estimated. These foods were excluded from further analyses.

Five of the 35 manufacturers responded when verifying these results: three confirmed that the estimated amounts were correct for margarine, filled milk chocolate bar with hazelnuts and hazelnut chocolate bar. Two confirmed that the estimated amounts were incorrect, namely for beef salad and tortellini. According to the manufacturer, beef salad contained $1 \mathrm{~g}$ of egg protein per portion instead of $0.5 \mathrm{~g}$ according to our estimation. For tortellini, the content of egg protein was $1.19 \mathrm{~g}$ per portion instead of $0.91 \mathrm{~g}$ per portion. The remaining 30 manufacturers either did not respond or responded but did not confirm or reject the amounts estimated and indicated that they were not willing to share the amount of allergenic protein of their products.

It was found that the actual or estimated amounts of allergenic protein varied widely in foods, and as expected, was highest in non-composite foods (Table 1). Of the noncomposite foods with milk, the highest amounts of milk protein per portion were found in skimmed milk $0.1 \%$, semi-skimmed milk $1.5 \%$, whole milk $3.5 \%$, buttermilk, and low-fat Gouda cheese: $9250 \mathrm{mg}, 8500 \mathrm{mg}, 8250 \mathrm{mg}, 7500 \mathrm{mg}$, and $6840 \mathrm{mg}$, respectively. The lowest amounts of milk protein per portion were observed for whipping cream, coffee creamer and butter: $230 \mathrm{mg}$, $50 \mathrm{mg}$, and $40 \mathrm{mg}$, respectively. Peanut butter yielded $3200 \mathrm{mg}$ peanut protein per portion. 


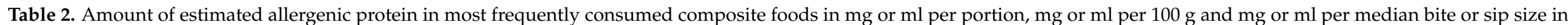
different age groups.

\begin{tabular}{|c|c|c|c|c|c|c|}
\hline Food & $\begin{array}{c}\text { Composite or } \\
\text { Non-Composite Food }\end{array}$ & $\begin{array}{l}\text { Amount of Protein } \\
\text { (mg or } \mathrm{ml} / \text { Portion) }\end{array}$ & $\begin{array}{l}\text { Amount of Protein } \\
(\mathrm{mg} \text { or } \mathrm{ml}) / \mathbf{1 0 0 g})\end{array}$ & $\begin{array}{l}\text { Amount of Protein (mg } \\
\text { or ml)/Median Bite or } \\
\text { Sip Size 2-3 Years }\end{array}$ & $\begin{array}{l}\text { Amount of Protein (mg } \\
\text { or ml)/Median Bite or } \\
\text { Sip Size 4-6 years }\end{array}$ & $\begin{array}{l}\text { Amount of Protein (mg } \\
\text { or ml)/Median Bite or } \\
\text { Sip Size 19-30 years) }\end{array}$ \\
\hline \multicolumn{7}{|c|}{ COW'S MILK } \\
\hline \multicolumn{7}{|c|}{ Bread and Crackers } \\
\hline Currant bread ${ }^{* *(J u m b o)}$ & Composite food & 590 & 1700 & 70 & 70 & 100 \\
\hline White bread (Jumbo *) & Composite food & 770 & 1700 & 30 & 30 & 100 \\
\hline \multicolumn{7}{|c|}{ Spread } \\
\hline $\begin{array}{l}\text { Chocolate hazelnut spread } \\
\text { (Nutella) }\end{array}$ & Composite food & 470 & 3160 & 30 & 30 & 80 \\
\hline \multicolumn{7}{|c|}{ Cake and Biscuits } \\
\hline $\begin{array}{l}\left.\text { Filled Biscuit (Biscuit fourré }{ }^{* *}\right) \\
\left(\text { Jumbo }^{*}\right)\end{array}$ & Composite food & 8.75 & 175 & $<10$ & $<10$ & $<10$ \\
\hline Syrup waffle (Jumbo *) & Composite food & 70 & 180 & $<10$ & $<10$ & $<10$ \\
\hline Waffle (Jumbo *) & Composite food & 90 & 180 & $<10$ & $<10$ & 20 \\
\hline $\begin{array}{l}\text { Eclair with whipped cream } \\
\left.\text { filling (Roomsoesje }{ }^{* *}\right)\left(\text { Jumbo }^{*}\right)\end{array}$ & Composite food & 190 & 1550 & $\mathrm{Nd}$ & $\mathrm{Nd}$ & $\mathrm{Nd}$ \\
\hline $\begin{array}{l}\text { Apple flan and crumble topping } \\
\left.\text { (Jumbo }{ }^{*}\right)\end{array}$ & Composite food & 430 & 430 & $\mathrm{Nd}$ & $\mathrm{Nd}$ & $\mathrm{Nd}$ \\
\hline Cake [19] & Composite food & 550 & 1830 & 70 & 70 & 160 \\
\hline $\begin{array}{l}\text { Cheesecake with fromage frais } \\
\left(\text { Dr. Oetker }{ }^{*}\right)\end{array}$ & Composite food & $2000-6000$ & $2000-6000$ & $\mathrm{Nd}$ & $\mathrm{Nd}$ & $\mathrm{Nd}$ \\
\hline \multicolumn{7}{|c|}{ Vegetables } \\
\hline Creamed spinach frozen (Iglo) & Composite food & 600 & 1250 & 50 & 250 & 130 \\
\hline \multicolumn{7}{|c|}{ Milk, Milk Products, Milk Replacers and Ice Cream } \\
\hline $\begin{array}{l}\text { Ice cream dairy, Cornetto Classic } \\
\text { (Ola) }\end{array}$ & Composite food & 882 & 1470 & $\mathrm{Nd}$ & $\mathrm{Nd}$ & $\mathrm{Nd}$ \\
\hline
\end{tabular}


Table 2. Cont.

\begin{tabular}{|c|c|c|c|c|c|c|}
\hline Food & $\begin{array}{c}\text { Composite or } \\
\text { Non-Composite Food }\end{array}$ & $\begin{array}{l}\text { Amount of Protein } \\
\text { (mg or ml/Portion) }\end{array}$ & $\begin{array}{l}\text { Amount of Protein } \\
(\mathrm{mg} \text { or } \mathrm{ml}) / \mathbf{1 0 0 g})\end{array}$ & $\begin{array}{l}\text { Amount of Protein (mg } \\
\text { or } \mathrm{ml}) / \text { Median Bite or } \\
\text { Sip Size 2-3 Years }\end{array}$ & $\begin{array}{l}\text { Amount of Protein }(\mathrm{mg} \\
\text { or } \mathrm{ml}) / \text { Median Bite or } \\
\text { Sip Size } 4-6 \text { years }\end{array}$ & $\begin{array}{l}\text { Amount of Protein (mg } \\
\text { or ml)/Median Bite or } \\
\text { Sip Size 19-30 years) }\end{array}$ \\
\hline $\begin{array}{l}\text { Vanilla custard full fat } \\
\text { (Friesland Campina *) }\end{array}$ & Composite food & 3600 & 2400 & 140 & 140 & 460 \\
\hline $\begin{array}{l}\text { Ice cream dairy, cream based } \\
\text { (Hertog) }\end{array}$ & Composite food & 3690 & 2460 & $\mathrm{Nd}$ & $\mathrm{Nd}$ & $\mathrm{Nd}$ \\
\hline $\begin{array}{c}\text { Baby Porridge vanilla } \\
\text { (Pyjama-papje **) (Nestlé) }\end{array}$ & Composite food & 3840 & 1920 & $\mathrm{Nd}$ & $\mathrm{Nd}$ & $\mathrm{Nd}$ \\
\hline \multicolumn{7}{|c|}{ Composite Meals } \\
\hline $\begin{array}{l}\text { Infant jarred food: Lasagna with } \\
\text { vegetables (Olvarit) }\end{array}$ & Composite food & 1100 & 550 & $\mathrm{Nd}$ & $\mathrm{Nd}$ & $\mathrm{Nd}$ \\
\hline Pancakes) [19] & Composite food & 1491 & 2130 & 90 & 60 & $\mathrm{Nd}$ \\
\hline \multicolumn{7}{|c|}{ Soup } \\
\hline $\begin{array}{l}\text { Chinese Tomato soup, canned } \\
\text { (Unox) }\end{array}$ & Composite food & 110 & 40 & $\mathrm{Nd}$ & $\mathrm{Nd}$ & $<10$ \\
\hline $\begin{array}{c}\text { Foam sweets banana flavor } \\
\text { (Bananen schuimpjes }{ }^{* *} \text { ) } \\
\text { (Haribo) }\end{array}$ & Composite food & 6 & $120^{*}$ & $\mathrm{Nd}$ & $\mathrm{Nd}$ & $\mathrm{Nd}$ \\
\hline Fudge Caramel Vanilla (Lonka) & Composite food & 80 & 1575 & $\mathrm{Nd}$ & $\mathrm{Nd}$ & $\mathrm{Nd}$ \\
\hline $\begin{array}{l}\text { Chocolate bar with hazelnuts } \\
\text { (Verkade) }\end{array}$ & Composite food & 180 & 3510 & 100 & 70 & 210 \\
\hline $\begin{array}{l}\text { Filled milk chocolate bar with } \\
\text { hazelnuts (BonBon Bloc Praliné } \\
\left.\text { milk }^{* *}\right)(\text { Cote d'Or) }\end{array}$ & Composite food & 650 & 4310 & 130 & 090 & 260 \\
\hline $\begin{array}{l}\text { Belgium chocolate (Zeevruchten } \\
\text { bonbon }{ }^{* *} \text { (Isaura) }\end{array}$ & Composite food & 650 & 4320 & 130 & 90 & 260 \\
\hline
\end{tabular}


Table 2. Cont.

\begin{tabular}{|c|c|c|c|c|c|c|}
\hline Food & $\begin{array}{c}\text { Composite or } \\
\text { Non-Composite Food }\end{array}$ & $\begin{array}{l}\text { Amount of Protein } \\
\text { (mg or ml/Portion) }\end{array}$ & $\begin{array}{l}\text { Amount of Protein } \\
(\mathrm{mg} \text { or } \mathrm{ml}) / 100 \mathrm{~g})\end{array}$ & $\begin{array}{l}\text { Amount of Protein (mg } \\
\text { or } \mathrm{ml}) / \text { Median Bite or } \\
\text { Sip Size 2-3 Years }\end{array}$ & $\begin{array}{l}\text { Amount of Protein }(\mathrm{mg} \\
\text { or } \mathrm{ml}) / \text { Median Bite or } \\
\text { Sip Size } 4-6 \text { years }\end{array}$ & $\begin{array}{l}\text { Amount of Protein (mg } \\
\text { or ml)/Median Bite or } \\
\text { Sip Size 19-30 years) }\end{array}$ \\
\hline \multicolumn{7}{|c|}{ Fat, Oil and Sauce } \\
\hline $\begin{array}{l}\text { Low-fat margarine (Gouda's } \\
\text { Glorie *) }\end{array}$ & Composite food & 4 & $80^{*}$ & $<10$ & $<10$ & $<10$ \\
\hline Tzatziki (Remia) & Composite food & 110 & 740 & $\mathrm{Nd}$ & $\mathrm{Nd}$ & 30 \\
\hline Gravy, powdered (Knorr) & Composite food & 110 & 700 & $\mathrm{Nd}$ & $\mathrm{Nd}$ & $\mathrm{Nd}$ \\
\hline Bechamel sauce [19] & Composite food & 1300 & 8680 & $\mathrm{Nd}$ & $\mathrm{Nd}$ & $\mathrm{Nd}$ \\
\hline \multicolumn{7}{|c|}{ Meat and Poultry } \\
\hline Hamburger (Mora) & Composite food & 780 & 1060 & 20 & 20 & 100 \\
\hline Ragout, beef, canned (Unox) & Composite food & 175 & 350 & $\mathrm{Nd}$ & $\mathrm{Nd}$ & $\mathrm{Nd}$ \\
\hline \multicolumn{7}{|c|}{ HEN'S EGG } \\
\hline \multicolumn{7}{|c|}{ Bread and Crackers } \\
\hline Round toast (Bolletje *) & Composite food & 5 & 50 & $\mathrm{Nd}$ & $\mathrm{Nd}$ & $\mathrm{Nd}$ \\
\hline \multicolumn{7}{|c|}{ Cake and Biscuits } \\
\hline Syrup waffle (Jumbo *) & Composite food & 3 & 6 & $<10$ & $<10$ & $<10$ \\
\hline Penny waffle (Jumbo *) & Composite food & 3 & 33 & $<10$ & $<10$ & $\mathrm{Nd}$ \\
\hline $\begin{array}{l}\text { Marzipan and chocolate cake } \\
\left(\text { Mergpijpje }^{* *}\right)\left(\text { Jumbo }^{*}\right)\end{array}$ & Composite food & 100 & 980 & $\mathrm{Nd}$ & $\mathrm{Nd}$ & $\mathrm{Nd}$ \\
\hline $\begin{array}{l}\text { Chocolate coated marsh mellow } \\
\quad\left(\text { Schuimzoenen }{ }^{* *}\right) \text { (Buys) }\end{array}$ & Composite food & 140 & 1400 & $\mathrm{Nd}$ & $\mathrm{Nd}$ & $\mathrm{Nd}$ \\
\hline $\begin{array}{l}\text { Eclair with whipped cream } \\
\left.\text { filling (Roomsoesje }{ }^{* *} \text { (Jumbo }{ }^{*}\right)\end{array}$ & Composite food & 220 & 1720 & $\mathrm{Nd}$ & $\mathrm{Nd}$ & $\mathrm{Nd}$ \\
\hline Cake [19] & Composite food & 390 & 1300 & 50 & 50 & 120 \\
\hline
\end{tabular}


Table 2. Cont.

\begin{tabular}{|c|c|c|c|c|c|c|}
\hline Food & $\begin{array}{c}\text { Composite or } \\
\text { Non-Composite Food }\end{array}$ & $\begin{array}{l}\text { Amount of Protein } \\
\text { (mg or ml/Portion) }\end{array}$ & $\begin{array}{l}\text { Amount of Protein } \\
(\mathrm{mg} \text { or } \mathrm{ml}) / 100 \mathrm{~g})\end{array}$ & $\begin{array}{l}\text { Amount of Protein (mg } \\
\text { or ml)/Median Bite or } \\
\text { Sip Size 2-3 Years }\end{array}$ & $\begin{array}{l}\text { Amount of Protein }(\mathrm{mg} \\
\text { or ml)/Median Bite or } \\
\text { Sip Size 4-6 years }\end{array}$ & $\begin{array}{l}\text { Amount of Protein (mg } \\
\text { or ml)/Median Bite or } \\
\text { Sip Size 19-30 years) }\end{array}$ \\
\hline $\begin{array}{c}\left.\text { Dutch sponge cake (Eierkoek }{ }^{* *}\right) \\
(\mathrm{AH})\end{array}$ & Composite food & 660 & 2210 & 90 & 90 & 200 \\
\hline Waffle (Jumbo *) & Composite food & 1970 & 3940 & 118 & 158 & 355 \\
\hline \multicolumn{7}{|c|}{ Pasta } \\
\hline Ravioli (Grand Italia) & Composite food & 1190 & 2380 & $\mathrm{Nd}$ & $\mathrm{Nd}$ & $\mathrm{Nd}$ \\
\hline Tortellini (Grand Italia) & Composite food & 1190 & 2380 & $\mathrm{Nd}$ & $\mathrm{Nd}$ & $\mathrm{Nd}$ \\
\hline \multicolumn{7}{|c|}{ Milk, Milk Products, Milk Replacers and Ice Cream } \\
\hline $\begin{array}{l}\text { Ice cream dairy, Cornetto Classic } \\
\text { (Ola) }\end{array}$ & Composite food & 3 & 5 & $\mathrm{Nd}$ & $\mathrm{Nd}$ & $\mathrm{Nd}$ \\
\hline \multicolumn{7}{|c|}{ Snacks, Meals } \\
\hline Beef salad (Johma) & Composite food & 50 & 30 & $\mathrm{Nd}$ & $\mathrm{Nd}$ & $\mathrm{Nd}$ \\
\hline \multicolumn{7}{|c|}{ Composite Dishes } \\
\hline Pancakes [19] & Composite food & 8610 & 12,300 & 492 & 369 & $\mathrm{Nd}$ \\
\hline \multicolumn{7}{|c|}{ Fat, Oil and Sauce } \\
\hline $\begin{array}{l}\text { Salad cream } 25 \% \text { oil (Slasaus **) } \\
\text { (Remia) }\end{array}$ & Composite food & 20 & 150 & $\mathrm{Nd}$ & $\mathrm{Nd}$ & $\mathrm{Nd}$ \\
\hline $\begin{array}{l}\text { Sauce for chips } 35 \% \text { oil } \\
\text { (Fritessaus }^{* *} \text { (Remia) }\end{array}$ & Composite food & 50 & 334 & $<10$ & 20 & 10 \\
\hline Mayonnaise (Remia) & Composite food & 130 & 840 & $<10$ & 500 & 30 \\
\hline \multicolumn{7}{|c|}{ PEANUT } \\
\hline \multicolumn{7}{|c|}{ Cake and Biscuits } \\
\hline Peanut cookie $($ Jumbo *) & Composite food & 630 & 6300 & 63 & 63 & $\mathrm{Nd}$ \\
\hline \multicolumn{7}{|c|}{ Snack Food } \\
\hline
\end{tabular}


Table 2. Cont

\begin{tabular}{|c|c|c|c|c|c|c|}
\hline Food & $\begin{array}{c}\text { Composite or } \\
\text { Non-Composite Food }\end{array}$ & $\begin{array}{l}\text { Amount of Protein } \\
\text { (mg or ml/Portion) }\end{array}$ & $\begin{array}{l}\text { Amount of Protein } \\
(\mathrm{mg} \text { or } \mathrm{ml}) / 100 \mathrm{~g})\end{array}$ & $\begin{array}{l}\text { Amount of Protein (mg } \\
\text { or } \mathrm{ml}) / \text { Median Bite or } \\
\text { Sip Size 2-3 Years }\end{array}$ & $\begin{array}{l}\text { Amount of Protein (mg } \\
\text { or ml)/Median Bite or } \\
\text { Sip Size 4-6 years }\end{array}$ & $\begin{array}{l}\text { Amount of Protein (mg } \\
\text { or ml)/Median Bite or } \\
\text { Sip Size 19-30 years) }\end{array}$ \\
\hline $\begin{array}{l}\text { Japanese rice cracker mix with } \\
\text { peanuts (Davis) }\end{array}$ & Composite food & 600 & 3020 & $\mathrm{Nd}$ & $\mathrm{Nd}$ & $\mathrm{Nd}$ \\
\hline Coated peanuts (Duyvis) & Composite food & 2720 & 13,610 & $\mathrm{Nd}$ & $\mathrm{Nd}$ & $\mathrm{Nd}$ \\
\hline \multicolumn{7}{|c|}{ Sweets and Chocolate } \\
\hline Candy bar, Snickers & Composite food & 1210 & 6050 & 180 & 180 & $\mathrm{Nd}$ \\
\hline M\&M's, chocolate with peanut & Composite food & 1160 & 5800 & $\mathrm{Nd}$ & $\mathrm{Nd}$ & $\mathrm{Nd}$ \\
\hline $\begin{array}{l}\text { Peanuts coated with milk } \\
\left.\text { chocolate (Chocopinda's }{ }^{* *}\right) \\
\left(\text { Jumbo }^{*}\right)\end{array}$ & Composite food & 1260 & 6300 & $\mathrm{Nd}$ & $\mathrm{Nd}$ & $\mathrm{Nd}$ \\
\hline \multicolumn{7}{|c|}{ Fats, Oils and Savory Sauces } \\
\hline Peanut sauce (Wijko) & Composite food & 1820 & 12,100 & 120 & 610 & 420 \\
\hline \multicolumn{7}{|c|}{ HAZELNUT } \\
\hline $\begin{array}{l}\text { Chocolate hazelnut spread } \\
\text { (Nutella) }\end{array}$ & Composite food & 270 & 1820 & 20 & 20 & 50 \\
\hline \multicolumn{7}{|c|}{ Cake and Biscuits } \\
\hline Penny waffle (Jumbo *) & Composite food & 7 & 70 & $<10$ & $<10$ & $\mathrm{Nd}$ \\
\hline \multicolumn{7}{|c|}{ Cereals } \\
\hline Muesli (Jumbo *) & Composite food & 60 & 140 & $\mathrm{Nd}$ & $\mathrm{Nd}$ & $<10$ \\
\hline \multicolumn{7}{|c|}{ Milk, Milk Products, Milk Replacers and Ice Cream } \\
\hline $\begin{array}{l}\text { Ice cream dairy, Cornetto Classic } \\
\text { (Ola) }\end{array}$ & Composite food & 168 & 280 & $\mathrm{Nd}$ & $\mathrm{Nd}$ & $\mathrm{Nd}$ \\
\hline \multicolumn{7}{|c|}{ Sugar, Sweets, Chocolate and Sweet Sauces } \\
\hline $\begin{array}{l}\text { Belgium chocolate (Zeevruchten } \\
\text { bonbon }{ }^{* *} \text { ) (Isaura) }\end{array}$ & Composite food & 380 & 2520 & 80 & 580 & $\mathrm{Nd}$ \\
\hline
\end{tabular}

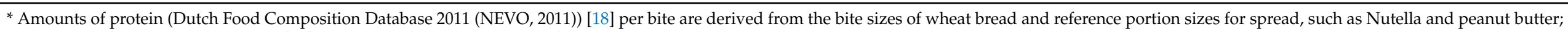

** Amounts of protein (NEVO, 2011) [18] are derived from a comparable food in the NEVO table; mg, milligram; ml, milliliter; g, gram; Nd, no data. 
Of the composite foods with milk (Table 2), the highest amounts of milk protein per portion were found in cheesecake, baby porridge, ice cream, vanilla custard and pancakes: up to $6000 \mathrm{mg}$, $3840 \mathrm{mg}$, $3690 \mathrm{mg}, 3600 \mathrm{mg}$, and $1491 \mathrm{mg}$, respectively. Relatively low amounts of milk protein were found in low-fat margarine, foam sweets banana flavor and filled biscuit, and creamer: $4 \mathrm{mg}, 6 \mathrm{mg}, 8 \mathrm{mg}$, and $75 \mathrm{mg}$ of milk protein per portion, respectively.

Of the composite foods with egg (Table 2), the highest amounts of eggprotein per portion were found in pancakes, waffles, ravioli and tortellini: $8610 \mathrm{mg}, 1970 \mathrm{mg}, 1190 \mathrm{mg}$ and $1190 \mathrm{mg}$, respectively. Low amounts of egg protein were found in round toast, syrup waffles, penny waffles and Cornetto ice cream: $5 \mathrm{mg}, 3 \mathrm{mg}, 3 \mathrm{mg}$, and $3 \mathrm{mg}$ of egg protein per portion, respectively.

Of the composite foods with peanut (Table 2), the amounts of peanut protein per portion varied between $630 \mathrm{mg}$ (peanut cookie) and $2720 \mathrm{mg}$ (coated peanuts).

Of the composite foods with hazelnut (Table 2), the amounts of hazelnut protein per portion varied between $380 \mathrm{mg}$ (Belgium bonbon) and $7 \mathrm{mg}$ (penny waffle).

\subsection{Comparison of the Estimated Amount of Allergenic Protein to ED10 and ED50}

The estimated amount of allergenic milk-, egg-, peanut- and hazelnut-protein per portion were compared to the ED10 and ED50 in children for objective symptoms as established in a Dutch population by Blom et al. [20]. Additionally, the estimated amount of peanut protein per portion was compared to the ED10 and ED50 in children and adults for objective and subjective symptoms by Klemans et al. [21].

- Milk

None of the selected composite or non-composite foods contained less estimated milk protein per portion than the ED10 (4.24 mg), except low-fat margarine. Nine foods contained less estimated milk protein per portion than the ED 50 (156 mg). The other foods contained higher estimated amounts.

- $\quad$ Egg

Four foods contained less estimated egg protein per portion than the ED10 (5.82 mg), while ten foods contained less estimated egg protein per portion than the ED50 (199 mg). All the other foods contained more estimated egg protein per portion.

- Peanut

None of the foods contained less estimated peanut protein per portion than the ED10 (4.42 $\mathrm{mg}$ ) by Blom [20], the ED10 in children $(18.6 \mathrm{mg})$ and in adults (13.7 $\mathrm{mg})$ by Klemans [21] or the ED50 in children $(67,3 \mathrm{mg})$ by Blom [20]. Only one food contained less peanut protein than the ED50 in adults $(821 \mathrm{mg})$ by Klemans [21].

- Hazelnut

None of the foods contained less estimated hazelnut protein per portion than the ED10 (1.38 mg) by Blom [20]. Two foods contained less estimated hazelnut protein per portion than the ED50 (80.6 mg) by Blom [20].

\subsection{Assessment of Bite and Sip Sizes in Different Age Groups}

Thirty foods were selected: 17 foods for the children 2-3 years of age, 17 foods for the children 4-6 years of age and 19 foods for the adults 19-30 years of age. Several foods were selected for more than one age group. In total, 71 participants were included (41 male $(57.7 \%) ; 30$ females $(42.3 \%))$.

In the 2-3-year-old age group, 18 toddlers participated ( 8 males, 10 females; median 3 years of age). A maximum of eight foods were tested in each child. The sip and bite sizes were close for all foods, except for soft drinks in which the largest median sip size was observed $(11 \mathrm{~mL})$ in contrast to milk, in which the smallest median intake was measured $(2.5 \mathrm{~mL})$. A large range in bite sizes was measured for pancakes (3.00-9.00 g). There were no significant differences between boys and girls in bite or sip sizes of the selected foods. 
In the age group of 4-6-year-old children, 39 children were included ( 28 males, 11 females; median 4 years). For each child a maximum of eight foods were tested (Table 3). The sip and bite sizes were similar for all foods. Between boys and girls, there was only a significant difference in bite size for pancakes $(p=0.008)$ [19].

Table 3. Median (IQR) bite and sip sizes in different age groups in grams or milliliters.

\begin{tabular}{|c|c|c|}
\hline Type of Food & $\begin{array}{l}\text { Median Weight or Volume in } \\
\text { Gram or ml }{ }^{*}(\text { IQR })^{*}\end{array}$ & Number of Participants \\
\hline \multicolumn{3}{|c|}{$2-3$ years of age } \\
\hline Wheat bread & $2.00(2.00-3.00)$ & 9 \\
\hline $\begin{array}{c}\text { Chocolate hazelnut spread, } \\
\text { Nutella* }\end{array}$ & 0.86 & n.d. \\
\hline Peanut butter * & 0.86 & n.d. \\
\hline Low-fat margarine * & 0.29 & n.d. \\
\hline Cheese* & 1.14 & n.d. \\
\hline Milk ** & $2.50(2.00-4.25)$ & 6 \\
\hline Boiled egg & $2.50(2.00-4.50)$ & 4 \\
\hline Biscuit (Maria biscuit) & $1.00(1.00-1.00)$ & 8 \\
\hline Crisps (Hamka's) & $0.50(0.25-0.60)$ & 5 \\
\hline Currant bread & $3.00(1.25-5.50)$ & 4 \\
\hline Pancake & $4.00(3.00-9.00)$ & 5 \\
\hline Snickers & $2.50(1.88-3.25)$ & 6 \\
\hline Soft drink (Taksi) ** & $11.00(8.00-12.25)$ & 6 \\
\hline Vanilla custard & $6.00(-)$ & 3 \\
\hline Cake batter & $2.00(1.00-3.50)$ & 5 \\
\hline Fried egg & $3.00(1.00-3.00)$ & 6 \\
\hline Creamed spinach & $4.00(-)$ & 2 \\
\hline Chicken nuggets & $2.00(1.75-3.50)$ & 6 \\
\hline Mayonnaise & $1.00(1.00-2.00)$ & 6 \\
\hline Milk chocolate & $2.50(1.00-4.00)$ & 10 \\
\hline \multirow[t]{2}{*}{ Muffin } & $3.00(1.00-4.50)$ & 9 \\
\hline & 4-6 years of age & \\
\hline Wheat bread & $2.00(1.00-2.00)$ & 17 \\
\hline Hazelnut spread * & 0.86 & n.d. \\
\hline Peanut butter * & 0.86 & n.d. \\
\hline Low-fat margarine * & 0.29 & n.d. \\
\hline Cheese* & 1.14 & n.d. \\
\hline Milk ** & $8.00(4.00-18.00)$ & 11 \\
\hline Boiled egg & $5.00(3.00-7.75)$ & 16 \\
\hline Biscuit & $1.00(1.00-2.00)$ & 19 \\
\hline Crisps (Hamka's chips) & $0.25(0.1875-0.5425)$ & 18 \\
\hline Currant bread & $3.00(2.00-4.00)$ & 15 \\
\hline Pancake & $3.00(2.00-5.25)$ & 18 \\
\hline Snickers & $3.00(2.00-4.00)$ & 16 \\
\hline Soft drink (Taksi) & $8.00(4.00-12.00)$ & 17 \\
\hline
\end{tabular}


Table 3. Cont.

\begin{tabular}{|c|c|c|}
\hline Type of Food & $\begin{array}{l}\text { Median Weight or Volume in } \\
\text { Gram or } \mathrm{ml}{ }^{*}(\mathrm{IQR}){ }^{*}\end{array}$ & Number of Participants \\
\hline Vanilla custard & $6.00(3.00-8.50)$ & 13 \\
\hline Cake batter & $1.00(0.50-1.00)$ & 7 \\
\hline Fried egg & $3.00(2.00-4.00)$ & 11 \\
\hline Cream spinach & $5.00(4.00-7.00)$ & 7 \\
\hline Chicken nuggets & $2.00(1.50-4.00)$ & 9 \\
\hline Mayonnaise & $0.50(0.50-1.00)$ & 9 \\
\hline Milk chocolate & $2.00(2.00-3.00)$ & 17 \\
\hline Muffin & $4.00(2.00-6.25)$ & 14 \\
\hline \multicolumn{3}{|c|}{ 19-30 years } \\
\hline Milk** & $32.00(24.00-58.75)$ & 14 \\
\hline Hardboiled egg & $12.00(7.75-16.50)$ & 14 \\
\hline Crisps (Hamka's chips) & $2.00(1.00-2.00)$ & 11 \\
\hline Soft drink (Taksi) & $36.00(30.70-43.75)$ & 14 \\
\hline Fried egg & $6.50(5.00-9.25)$ & 14 \\
\hline Cream spinach & $10.00(8.50-13.00)$ & 14 \\
\hline Muffin & $9.00(6.00-11.25)$ & 14 \\
\hline Soft drink (Rivella) & $31.50(27.25-50.75)$ & 14 \\
\hline Canned Soup & $9.00(8.00-10.00)$ & 12 \\
\hline Cappuccino & $23.50(14.50-37.50)$ & 14 \\
\hline White bread & $6.00(3.75-8.25)$ & 14 \\
\hline Hazelnut spread * & 2.57 & n.d. \\
\hline Peanut butter * & 2.57 & n.d. \\
\hline Low-fat margarine * & 0.86 & n.d. \\
\hline Cheese * & 3.43 & n.d. \\
\hline Yogurt with muesli (Cruesli) & $19.00(14.00-21.25)$ & 14 \\
\hline Potato croquette & $5.50(4.75-8.25)$ & 14 \\
\hline Schnitzel & $9.00(8.50-10.00)$ & 14 \\
\hline Sate sauce & $3.50(2.00-6.00)$ & 14 \\
\hline Nougat & $5.50(4.75-7.25)$ & 14 \\
\hline Belgium chocolate & $6.00(3.75-10.00)$ & 14 \\
\hline Spiced biscuit & $3.00(3.00-5.00)$ & 14 \\
\hline
\end{tabular}

n.d.: not done. ${ }^{*}$ Amounts of protein (NEVO, 2011) (12) are derived from the bite sizes of wheat bread and reference portion sizes for spreads, such as Nutella and peanut butter (17) ${ }^{* *} 1 \mathrm{~g}$ is considered equivalent to 1 milliliter.

In the age group 19-30 years, fourteen adults were included (5 males, 9 females; median age 22 years). There were large differences in the bite and sip sizes for the foods within this age group (Table 3). The largest interquartile range (IQR) was observed for milk $(24.00-58.75 \mathrm{~mL})$. Between men and women, significant differences in bite and sip sizes were found for eleven other foods and drinks ( $p$ values 0.001 to 0.042 ). 


\subsubsection{Differences between the Different Age Groups}

In the 2-3-year-old children, the bite and sip sizes for wheat bread and mayonnaise were significantly larger than those in 4-6-year-old children $(p=0.029$ and $p=0.012)$, whereas 4-6-year-old children had significantly larger sip sizes for milk $(p=0.010)$.

For the foods tested in all age groups, the bite and sip sizes of the 19-30-year-old adults were significantly larger compared to the 2-3-year-old children and 4-6-year-old children for all foods.

\subsubsection{Amount of Protein per Bite or Sip}

In Tables 1 and 2 it is shown that a single bite or sip of many foods contains sufficient amounts of allergenic protein to elicit an allergic reaction.

\section{Discussion}

This study aimed to test the feasibility of estimating the amount of allergenic protein in frequently consumed foods, as estimated per $100 \mathrm{~g}$, per portion and per bite and sip sizes in different age groups for improved diagnosis and management of food allergies. For noncomposite foods, the amount of allergenic protein could easily be derived from the label or food composition tables, as all the protein was delivered by one allergenic ingredient. For composite foods we showed that it is very hard to obtain detailed information about the amount of allergenic protein. Through a lot of effort, the allergenic protein content of many composite foods may at best be estimated, however, true amounts of allergenic protein values may be somehow different.

For most composite foods depicted in Table 2, the amounts of allergenic protein are estimates rather than established amounts of protein. Based on our estimations, most composite foods contain less than 2-3 g of allergenic protein, except a few products that contain higher amounts such as cheesecake, baby porridge, ice cream, vanilla custard and pancakes.

For 19/70 (27\%) of the composite foods, the amount of allergenic protein could not be estimated due to lack of detailed information on the label or lack of information from the manufacturer.

Four manufacturers provided us the required protein amounts of four foods $(6 \%)$. For the other $47 / 70(67 \%)$ composite foods, the amount of allergenic protein could be estimated using a non-validated method. Only $2 / 70(3 \%)$ of the composite foods fully disclosed the amount of all allergenic ingredients (Nutella and peanut butter).

If detailed data on the amount of allergenic ingredients were provided by the manufacturer, these data could increase the quality of the diagnosis and management of patients with food allergies. First, full disclosure of not only the presence of allergens but also the amount of allergenic protein on the labels would allow for quantitative risk assessment in diet history and diagnosis. The health care professional could better assess how much allergenic protein is ingested prior to the allergic reaction. These data would help to establish the sensitivity of the patient for the allergenic food in question and, if necessary, sustain decision-making on extra safety measures during oral food challenges in highly sensitive patients. In addition, patients having reacted (severely) to small amounts in history will receive stringent dietary avoidance advice.

Second, detailed data on the amount of allergenic ingredients would support the decision-making for epinephrine auto-injector prescription in clinically sensitive patients.

Third, detailed data on the amount of allergenic ingredients would enable individually tailored dietary advice in food allergic patients. It would allow patients who had a mild reaction to try higher doses in oral food challenge tests to safely introduce foods with small amounts into their diet well below their thresholds. This could include the use of foods containing precautionary labeling, such as "may contain traces of ... " [8] or foods containing small amounts of an allergen listed in the ingredient list. This information would allow the dietitian or patient to select foods with allergenic protein below their thresholds to expand the diet. Patients tolerating baked milk and baked egg could introduce products 
with baked egg and milk into their diets. Lastly, milk and egg ladders, practical tools developed by dietitians to introduce foods at home [22], could be adapted based on the amount of allergen listed on the label.

However, due to a lack of detailed information on the label, the estimations in this study on the amount of allergenic protein do not allow for detailed advice in everyday clinical practice. We therefore encourage companies to disclose the amount of common allergenic foods on their labels.

We do not expect that improved quantitative risk assessment in dietary history will precisely predict the threshold dose during an oral challenge, as exposure in daily life occurs in uncontrolled conditions. Previous studies have shown a lack of correlation between the severity of reactions at home and thresholds or severity during oral food challenges $[6,13,14]$. This may be due to an incomplete diet history with a lack of data on the exact amount of ingested allergenic food, because thresholds in oral food challenges vary over time and because of co-factors such as sleep deprivation and physical exercise $[13,14]$.

For clinical relevance we compared the amount of estimated allergenic protein per portion with the ED10 and ED50 for allergens as established by several authors [20,21] in the Dutch population. For milk, only one food contained less estimated allergenic protein per portion when comparing the amount of milk in foods to the ED10 for milk; for egg this was found for four foods, while for peanut and hazelnut none of the foods contained less than the ED10 [20,21]. This means that, theoretically, all the other foods will provoke allergic reactions in allergic patients who belong to the $10 \%$ most clinically sensitive individuals.

When comparing allergenic protein contents with the ED50, nine foods containing milk, ten foods containing egg, no foods containing peanut and two foods containing hazelnut had allergenic amounts per portion below the ED50 in children. Thus, when taking a diet history, inconsistent reactions may be explained by low amounts of allergenic protein in food, except for peanut. This is even more true when only one of a few bites or sips are taken from the food instead of a full portion.

This study showed a clear difference in bite or sip sizes between the different age groups. As expected, the median bite size increases with age. This difference was significant when comparing the adults with the two younger age groups. We also observed 19-30-year-old men having a larger bite and sip sizes for all types of food than women. We showed that a single bite or sip of many foods contain sufficient amounts of allergenic protein to elicit an allergic reaction.

In the literature, there are some data available about bite and sip sizes, however most studies are performed in adults and in obese versus lean study participants to study the effects of portion size and hunger or satiety on bite or sip sizes [23-26]. Bite sizes increase with increasing portion size $[23,25]$ and body mass index $[24,26]$. In our study, regular portions were administered and none of the study participants were extremely obese. Bite and sip size in men were larger than in women [23-25], as was found in our study. Our data on bite and sip sizes in both children and adults may further enhance the assessment of the intake of allergenic protein consumed.

Our study has several limitations. We used a non-validated method to assess the amounts of allergenic protein in composite foods. We are not aware of a validated approach, and quantitative measurement of allergenic protein in foods was beyond the scope of this study. We also did not use a power analysis to determine the number of study participants for bite and sip sizes. Therefore, the study participants we used to study bite and sip sizes may not be representative for the different age groups. Bite and sip sizes should therefore be interpreted with caution.

\section{Conclusions}

In conclusion, in everyday practice it is hard to obtain detailed and reliable information about the amount of allergenic protein incorporated in composite foods. Yet, this study provides some insight into the estimated amount of allergenic protein in a large number 
of commonly consumed foods per portion, per $100 \mathrm{~g}$ and also per bite or sip size in the Netherlands, as established using a non-validated method. Diet history may be inconsistent in less sensitive patients as they may not react to foods containing low amounts of allergenic protein. In contrast, a single bite or sip can contain sufficient amount of an allergenic protein to elicit an allergic reaction. Bite and sip sizes increased with age. Disclosure of the amount of allergenic protein on labels would improve quantitative risk assessment in diet history in clinical practice, as well as dietary management of food allergies by allowing patients to introduce foods into their diet that they tolerate.

Author Contributions: Conceptualization, B.V.-B.; methodology, B.V.-B., A.S. and I.P.; formal analysis, M.K., A.C. and I.P.; writing, M.K. and A.C.; B.V.-B., A.S. and I.P., supervision, B.V.-B. and I.P. All authors have read and agreed to the published version of the manuscript.

Funding: This research received no external funding.

Institutional Review Board Statement: The study was conducted according to the guidelines of the Declaration of Helsinki, and ethical review and approval were waived for this study, according to the Dutch Law.

Informed Consent Statement: Informed consent was obtained from the parents of the children and by the adult study participants. Additionally, children and adults had to be willing to take a bite or sip from the selected foods voluntarily. The schools and preschool gave their consent for this study, too.

Data Availability Statement: The data presented in this study are available on request from the corresponding author. A data sharing agreement will be requested.

Acknowledgments: The authors would like to thank the study participants, the schools and the preschool for their willingness to participate in the involved studies. We thank Isabel Skypala for her critical comments on the manuscript.

Conflicts of Interest: The authors declare no conflict of interest.

\section{References}

1. Boyce, J.A.; Assa'ad, A.; Burks, W.A.; Jones, S.M.; Sampson, H.A.; Wood, R.A.; Plaut, M.; Cooper, S.F.; Fenton, M.J.; Arshad, S.H.; et al. Guidelines for the diagnosis and management of food allergy in the United States: Report of the NIAID-sponsored expert panel. J. Allergy Clin. Immunol. 2010, 126, S1-S58. [CrossRef] [PubMed]

2. Muraro, A.; Werfel, T.; Hoffmann-Sommergruber, K.; Roberts, G.A.; Beyer, K.; Bindslev-Jensen, C.; Cardona, V.; Dubois, A.; Dutoit, G.; Eigenmann, P.; et al. EAACI food allergy and anaphylaxis guidelines: Diagnosis and management of food allergy. Allergy 2014, 69, 1008-1025. [CrossRef] [PubMed]

3. Skypala, I.J.; Venter, C.; Meyer, R.; de Jong, N.W.; Fox, A.T.; Groetch, M.; Elberink, J.N.O.; Sprikkelman, A.B.; Diamandi, L.; Diamandi, L.; et al. The development of a standardised diet history tool to support the diagnosis of food allergy. Clin. Transl. Allergy 2015, 5, 7. [CrossRef] [PubMed]

4. Eigenmann, P.; Atanaskovic-Markovic, M.; Hourihane, J.O.; Lack, G.; Lau, S.; Matricardi, P.M.; Muraro, A.; Baranova, L.N.; Nieto, A.; Papadopoulos, N.G.; et al. Testing children for allergies: why, how, who and when. Pediatr. Allergy Immunol. 2013, 24, 195-209. [CrossRef]

5. Turner, P.J.; Baumert, J.L.; Beyer, K.; Boyle, R.J.; Chan, C.-H.; Clark, A.; Crevel, R.W.R.; DunnGalvin, A.; Fernández-Rivas, M.; Gowland, M.H.; et al. Can we identify patients at risk of life-threatening allergic reactions to food? Allergy 2016, 71, 1241-1255. [CrossRef] [PubMed]

6. Van der Zee, T.; Dubois, A.; Kerkhof, M.; van der Heide, S.; Vlieg-Boerstra, B.J. The eliciting dose of peanut in double-blind, placebo-controlled food challenges decreases with increasing age and specific IgE in children and young adults. J. Allergy Clin. Immunol. 2011, 128, 1031-1036. [CrossRef] [PubMed]

7. Dubois, A.E.J.; Turner, P.J.; Hourihane, J.; Ballmer-Weber, B.; Beyer, K.; Chan, C.H.; Gowland, M.H.; O’Hagan, S.; Regent, L.; Remington, B.; et al. How does dose impact on the severity of food-induced allergic reactions, and can this improve risk assessment for allergenic foods? Report from an ILSI Europe Food Allergy Task Force Expert Group and Workshop. Allergy 2018, 73, 1383-1392. [CrossRef] [PubMed]

8. Brough, H.A.; Turner, P.J.; Wright, T.; Fox, A.T.; Taylor, S.L.; Warner, J.O.; Lack, G. Dietary management of peanut and tree nut allergy: What exactly should patients avoid? Clin. Exp. Allergy 2015, 45, 859-871. [CrossRef] [PubMed]

9. The European Parliament and the Council of the European Union. REGULATION (EU) No 1169/2011 OF THE EUROPEAN PARLIAMENT AND OF THE COUNCIL of 25 October 2011. Available online: http:/ / eur-lex.europa.eu/LexUriServ/LexUriServ. do?uri=OJ:L:2011:304:0018:0063:EN:PDF (accessed on 11 October 2014). 
10. Mills, E.N.C.; Adel-Patient, K.; Bernard, H.; De Loose, M.; Gillard, N.; Huet, A.-C.; Larré, C.; Nitride, C.; Pilolli, R.; Tranquet, $\mathrm{O}$; et al. Detection and quantification of allergens in foods and minimum eliciting doses in food-allergic individuals (ThRAll). J. AOAC Int. 2019, 102, 1346-1353. [CrossRef] [PubMed]

11. Raposo, A.; Pérez, E.; Tinoco de Faria, C.; Carrascosa, C. Allergen management as a key issue in food safety. In Food Safety and Protection; Ravishankar Rai, V., Bai, J.A., Eds.; CRC Press: Boca Raton, FL, USA, 2017; pp. 195-242.

12. Sampson, H.A.; Van Wijk, R.G.; Bindslev-Jensen, C.; Sicherer, S.H.; Teuber, S.S.; Burks, A.W.; Dubois, A.E.; Beyer, K.; Eigenmann, P.A.; Spergel, J.M.; et al. Standardizing double-blind, placebo-controlled oral food challenges: American Academy of Allergy, Asthma \& Immunology_European Academy of Allergy and Clinical Immunology PRACTALL consensus report. J. Allergy Clin. Immunol. 2012, 130, 1260-1274. [CrossRef] [PubMed]

13. Glaumann, S.; Nopp, A.; Johansson, S.G.O.; Borres, M.P.; Nilsson, C. Oral peanut challenge identifies an allergy but the peanut allergen threshold sensitivity is not reproducible. PLoS ONE 2013, 8, e53465. [CrossRef]

14. Dua, S.; Ruiz-Garcia, M.; Bond, S.; Durham, S.R.; Kimber, I.; Mills, C.; Roberts, G.; Skypala, I.; Wason, J.; Ewan, P.; et al. Effect of sleep deprivation and exercise on reaction threshold in adults with peanut allergy: A randomized controlled study. J. Allergy Clin. Immunol. 2019, 144, 1584-1594.e2. [CrossRef] [PubMed]

15. Leonard, S.A.; Caubet, J.C.; Kim, J.S.; Groetch, M.; Nowak-Wegrzyn, A. Baked milk- and egg-containing diet in the management of milk and egg allergy. J. Allergy Clin. Immunol. Pract. 2015, 3, 13-23. [CrossRef] [PubMed]

16. Van Rossum, C.T.M.; Fransen, H.P.; Verkaik-Kloosterman, J.; Buurma-Rethans, E.J.M.; Ocke, M.C. RIVM Rapport Dutch National Food Consumption Survey 2007-2010: Diet of Children and Adults Aged 7 to 69 Years. 2011. Available online: https:/ / www.rivm.nl/bibliotheek/rapporten/350050006.pdf (accessed on 15 September 2013).

17. National Institute for Public Health and the Environment. Food Consumption Survey of Dutch Children (Users and Total Population) Age 2-6 Years. Available online: https:/ / www.rivm.nl/en/dutch-national-food-consumption-survey/overviewsurveys/young-children-2-6-years-2005-2006 (accessed on 15 September 2013).

18. National Institute for Public Health and the Environment. NEVO-online. Available online: http://nevo-online.rivm.nl/ (accessed on 30 January 2013).

19. Blommestein, I.; van Eijndhoven, A.; van Mill, J. Kook Ook, 8th ed.; VBK Media: Utrecht, The Netherlands, 2008.

20. Blom, W.M.; Vlieg-Boerstra, B.J.; Kruizinga, A.G.; Van Der Heide, S.; Houben, G.F.; Dubois, A.E.J. Threshold dose distributions for 5 major allergenic foods in children. J. Allergy Clin. Immunol. 2013, 131, 172-179. [CrossRef] [PubMed]

21. Klemans, R.J.B.; Blom, W.M.; van Erp, F.C.; Masthoff, L.J.N.; Rubingh, C.M.; van der Ent, C.K.; Bruijnzeel-Koomen, C.A.F.M.; Houben, G.F.; A Pasmans, S.G.M.; Meijer, Y.; et al. Objective eliciting doses of peanut-allergic adults and children can be combined for risk assessment purposes. Clin. Exp. Allergy 2015, 45, 1237-1244. [CrossRef] [PubMed]

22. Athanasopoulou, P.; Deligianni, E.; Dean, T.; Dewey, A.; Venter, C. Use of baked milk challenges and milk ladders in clinical practice: a worldwide survey of healthcare professionals. Clin. Exp. Allergy 2017, 47, 430-434. [CrossRef] [PubMed]

23. Burger, K.S.; Fisher, J.O.; Johnson, S.L. Mechanisms behind the portion size effect: Visibility and bite size. Obesity 2011, 19, 546-551. [CrossRef] [PubMed]

24. Hill, S.W.; McCutcheon, M.B. Contributions of obesity, gender, hunger, food preference, and body size to bite size, bite speed, and rate of eating. Appetite 1984, 5, 73-83. [CrossRef]

25. Forde, C.; Van Kuijk, N.; Thaler, T.; De Graaf, C.; Martin, N. Oral processing characteristics of solid savoury meal components, and relationship with food composition, sensory attributes and expected satiation. Appetite 2013, 60, 208-219. [CrossRef] [PubMed]

26. Hübel, R.; Laessle, R.; Lehrke, S.; Jass, J. Laboratory measurement of cumulative food intake in humans: Results on reliability. Appetite 2006, 46, 57-62. [CrossRef] [PubMed] 